\title{
Some Dimensional Results of a Class of Homogeneous Moran Sets
}

\author{
Jingru Zhang, ${ }^{1}$ Yanzhe Li $\mathbb{D}^{2}$, and Manli Lou ${ }^{3}$ \\ ${ }^{1}$ School of Mathematical Sciences, South China Normal University, Guangzhou 510631, China \\ ${ }^{2}$ College of Mathematics and Information Science, Guangxi Center for Mathematical Research, Guangxi University, \\ Nanning 530004, China \\ ${ }^{3}$ Department of Mathematics, Guangdong Polytechnic Normal University, Guangzhou 510665, China
}

Correspondence should be addressed to Yanzhe Li; lyzkbm@163.com

Received 1 May 2021; Accepted 26 June 2021; Published 7 July 2021

Academic Editor: Ji Gao

Copyright (C) 2021 Jingru Zhang et al. This is an open access article distributed under the Creative Commons Attribution License, which permits unrestricted use, distribution, and reproduction in any medium, provided the original work is properly cited.

In this paper, we construct a class of special homogeneous Moran sets: $\left\{m_{k}\right\}$-quasi-homogeneous perfect sets, and obtain the Hausdorff dimension of the sets under some conditions. We also prove that the upper box dimension and the packing dimension of the sets can get the maximum value of the homogeneous Moran sets under the condition $\sup _{k \geq 1} m_{k}<\infty$ and estimate the upper box dimension of the sets in two cases.

\section{Introduction}

The fractal dimensions of the Moran sets have been studied by many authors and close connected with many subjects, such as the multifractals (see [1-3]), the quasiconformal mappings (see $[4,5]$ ), the ergodic theory (see [6]), and the number theory (see [7]). In these applications, the homogeneous Moran sets play an important role. There are many classical results about the fractal dimensions of the homogeneous Moran sets (see $[8,9]$ ), but Wen et al. $[8,9]$ only showed the maximal values and the minimal values of the Hausdorff dimension, the upper box dimension, and the packing dimension (see [10]) of the family of the homogeneous Moran sets, and we need to get some more exact expressions of the fractal dimensions for some special homogeneous Moran sets. Wen and $\mathrm{Wu}$ [11] constructed a class of homogeneous Moran sets by the gaps, which are called homogeneous perfect sets, and obtained the Hausdorff dimension of the homogeneous perfect sets under some conditions. Later, Wang and $\mathrm{Wu}$ [12] obtained the upper box dimension and the packing dimension of the homogeneous perfect sets under some conditions. $\mathrm{Hu}[13,14]$ constructed a special kind of homogeneous Moran sets which is called $\left\{m_{k}\right\}$-quasi-homogeneous Cantor sets by the connected components and the gaps and obtained its Hausdorff dimension, upper box dimension, and packing dimension.

In this paper, a class of special homogeneous Moran sets, which is called $\left\{m_{k}\right\}$-quasi-homogeneous perfect sets, is constructed by the connected components and the gaps, and several results about the fractal dimensions of the sets are obtained, which extend the results in $[11,12,14]$ under some conditions.

This paper is organized as follows. In Section 2, we give the definitions of the homogeneous Moran sets, the homogeneous perfect sets, and the $\left\{m_{k}\right\}$-quasi-homogeneous perfect sets. Our main results are stated in Section 3. Section 4 gives the proofs of our theorems.

\section{Preliminaries}

First, we recall the definition of homogeneous Moran sets. Let $\left\{n_{k}\right\}_{k \geq 1}$ be a sequence of positive integers and $\left\{c_{k}\right\}_{k \geq 1}$ be a sequence of positive real numbers, such that $n_{k} \geq 2$, $0<c_{k}<1$ and $n_{k} c_{k}<1$, for any $k \geq 1$. Let $D_{k}=$ $\left\{\sigma_{1} \sigma_{2} \ldots \sigma_{k}: 1 \leq \sigma_{j} \leq n_{j}, 1 \leq j \leq k\right\}$, for any $k \geq 1$, and let $D_{0}=$ $\{\varnothing\}$ and $D=\cup_{k=0}^{\infty} D_{k}$. If $\sigma=\sigma_{1} \sigma_{2} \ldots \sigma_{k} \in D_{k} \quad$ and $1 \leq j \leq n_{k+1}$, let $\sigma * j=\sigma_{1} \sigma_{2} \ldots \sigma_{k} j \in D_{k+1}$. 
Definition 1 (homogeneous Moran sets, (see [8]). Let $I \subset \mathbb{R}$ be a nonempty closed interval and $\mathscr{F}=\left\{I_{\sigma}: \sigma \in D\right\}$ be a collection of closed subintervals of $I$. We say that $\mathscr{F}$ has homogeneous Moran structure if it satisfies

(i) $I_{\varnothing}=I$

(ii) For any $k \geq 0, \sigma \in D_{k}, I_{\sigma * 1}, I_{\sigma * 2}, \ldots, I_{\sigma * n_{k+1}}$ are subintervals of $I_{\sigma}$, and the interiors of $I_{\sigma * i}$ and $I_{\sigma * j}$ are disjoint for any $i \neq j$

(iii) For any $k \geq 1, \sigma \in D_{k-1}$, and $1 \leq j \leq n_{k}$, we have $\left(\left|I_{\sigma * j}\right| /\left|I_{\sigma}\right|\right)=c_{k}$, where $|A|$ denotes the diameter of the set $A \subset \mathbb{R}$

Let

$$
E=E(\mathscr{F}):=\bigcap_{k \geq 1} \bigcup_{\sigma \in D_{k}} I_{\sigma} .
$$

Then, we call $E$ a homogeneous Moran set satisfying $\left(I,\left\{n_{k}\right\},\left\{c_{k}\right\}\right)$ and denote by $\mathscr{M}\left(I,\left\{n_{k}\right\},\left\{c_{k}\right\}\right)$ the class of all homogeneous Moran sets associated with $I,\left\{n_{k}\right\}$, and $\left\{c_{k}\right\}$.

Let $\mathscr{F}_{k}=\left\{I_{\sigma}: \sigma \in D_{k}\right\}$; then, $\mathscr{F}=\cup_{k \geq 0} \mathscr{F}_{k}$ and any $I_{\sigma}$ in $\mathscr{F}_{k}$ is called a $k$-order basic interval of $E$. Without loss of generality, we set $I=[0,1]$ and assume that $I_{\sigma * 1}, \ldots, I_{\sigma * n_{k}}$ are located from left to right for any $k \geq 1$ and any $\sigma \in D_{k-1}$.

Based on the basic intervals and the gaps, Wen and $\mathrm{Wu}$ [11] constructed a special kind of homogeneous Moran sets: homogeneous perfect sets.

Definition 2 (homogeneous perfect sets, (see [11]). Let $E \in \mathscr{M}\left(I,\left\{n_{k}\right\},\left\{c_{k}\right\}\right)$, and we say that $\mathcal{J}=\left\{I_{\sigma}: \sigma \in D\right\}$ satisfies homogeneous perfect structure if it satisfies the following: there exists a sequence of real numbers $\left\{\eta_{k, j}: k \geq 1,0 \leq j \leq n_{k}\right\}$ such that, for any $k \geq 1,0 \leq j \leq n_{k}$, we have $\eta_{k, j} \geq 0$, and for any $k \geq 0, \sigma \in D_{k}$, and $1 \leq i \leq n_{k+1}-1$, we have $\min \left(I_{\sigma * 1}\right)-\min \left(I_{\sigma}\right)=\eta_{k+1,0}, \quad \min \left(I_{\sigma *(i+1)}\right)-$ $\max \left(I_{\sigma * i}\right)=\eta_{k+1, i}$, and $\max \left(I_{\sigma}\right)-\max \left(I_{\sigma * n_{k+1}}\right)=\eta_{k+1, n_{k+1}}$. Then, we call $E=E(\mathscr{J})=E\left(I,\left\{n_{k}\right\},\left\{c_{k}\right\},\left\{\eta_{k, j}\right\}\right):=$ $\cap_{k \geq 1} \cup_{\sigma \in D_{k}} I_{\sigma}$ a homogeneous perfect set satisfying $\left(I,\left\{n_{k}\right\},\left\{c_{k}\right\},\left\{\eta_{k, j}\right\}\right)$ and denote by $\mathscr{J}\left(I,\left\{n_{k}\right\},\left\{c_{k}\right\},\left\{\eta_{k, j}\right\}\right)$ the class of all homogeneous perfect sets associated with $I,\left\{n_{k}\right\},\left\{c_{k}\right\},\left\{\eta_{k, j}\right\}$.

Based on the connected components of the basic intervals and their gaps, we can construct a special class of homogeneous Moran sets: $\left\{m_{k}\right\}$-quasi-homogeneous perfect sets.

Definition 3 ( $\left\{m_{k}\right\}$-quasi-homogeneous perfect sets). Let $E \in \mathscr{M}\left(I,\left\{n_{k}\right\},\left\{c_{k}\right\}\right)$, if for all $k \geq 1$ and $\sigma \in D_{k-1}$, the $k$-order basic intervals $I_{\sigma * 1}, I_{\sigma * 2}, \ldots, I_{\sigma * n_{k}}$ arbitrarily connect forming $m_{k}\left(1 \leq m_{k} \leq n_{k}\right)$ connected components (denote by $J_{\sigma * 1}, J_{\sigma * 2}, \ldots, J_{\sigma * m_{k}}$, which are called $k$-order connected components). We say that $\mathscr{I}=\left\{I_{\sigma}: \sigma \in D\right\}$ satisfies homogeneous perfect structure if it satisfies

(i) $I_{\varnothing}=J=I$

(ii) There exists a sequence of real numbers $\left\{\eta_{k, l}^{*}: k \geq 1,0 \leq l \leq m_{k}\right\}$ such that, for any $k \geq 1,0 \leq l \leq$ $m_{k}$, we have $\eta_{k, j}^{*} \geq 0$ and $\min _{1 \leq l \leq m_{k}-1} \eta_{k, l}^{*}>0$, and for any $k \geq 0, \sigma \in D_{k}$, and $1 \leq i \leq m_{k+1}-1$, we have $\min \left(J_{\sigma * 1}\right)-\min \left(I_{\sigma}\right)=\eta_{k+1,0}^{*}, \min \left(J_{\sigma *(i+1)}\right)-$ $\max \left(J_{\sigma * i}\right)=\eta_{k+1, i}^{*}$, and $\max \left(I_{\sigma}\right)-\max \left(J_{\sigma * m_{k+1}}\right)=$ $\eta_{k+1, m_{k+1}}^{*}$

Let $E=E(\mathscr{I})=E\left(I, J,\left\{n_{k}\right\},\left\{m_{k}\right\},\left\{c_{k}\right\},\left\{\eta_{k, l}^{*}\right\}\right):=\cap_{k \geq 1}$ $\cup_{\sigma \in D_{k}} I_{\sigma}$; then, we call $E$ a $\left\{m_{k}\right\}$-quasi-homogeneous perfect set satisfying $\left(I, J,\left\{n_{k}\right\},\left\{c_{k}\right\},\left\{\eta_{k, l}^{*}\right\}\right)$ and denote by $\mathcal{J}^{*}\left(I, J,\left\{n_{k}\right\},\left\{m_{k}\right\},\left\{c_{k}\right\},\left\{\eta_{k, l}^{*}\right\}\right)$ the class of all $\left\{m_{k}\right\}$-quasihomogeneous perfect sets associated with $I, J,\left\{n_{k}\right\}$, $\left\{c_{k}\right\},\left\{\eta_{k, l}^{*}\right\}$. For any $\sigma \in D_{k-1}, \quad 1 \leq j \leq m_{k}$, denote by $\sharp\left\{i: I_{\sigma * i} \subset J_{\sigma * j}\right\}$ the number of the $k$-order basic intervals contained in the $k$-order connected components $J_{\sigma * j}$.

Remark 1. Let $E \in \mathcal{J}^{*}\left(I, J,\left\{n_{k}\right\},\left\{m_{k}\right\},\left\{c_{k}\right\},\left\{\eta_{k, l}^{*}\right\}\right)$; then, we have

(i) If $m_{k}=n_{k}$, for any $k \geq 1$, then $E$ is a homogeneous perfect set and $E \in \mathscr{J}\left(I,\left\{n_{k}\right\},\left\{c_{k}\right\},\left\{\eta_{k, l}\right\}\right)$, where $\eta_{k, l}=\eta_{k, l}^{*}$, for any $k \geq 1,0 \leq l \leq m_{k}$

(ii) If $m_{k}=n_{k}, \eta_{k, 0}^{*}=\eta_{k, m_{k}}^{*}=0$, and $\eta_{k, 1}^{*}=\cdots=\eta_{k, m_{k}-1}^{*}$, for any $k \geq 1$, then $E$ is a homogeneous Cantor set [8]

\section{Main Results}

Let

$$
\begin{aligned}
& s_{*}=\liminf _{k \longrightarrow \infty} \frac{\log n_{1} n_{2}, \ldots, n_{k}}{-\log c_{1} c_{2}, \ldots, c_{k}}, \\
& s^{*}=\limsup _{k \longrightarrow \infty} \frac{\log n_{1} n_{2}, \ldots, n_{k}}{-\log c_{1} c_{2}, \ldots, c_{k}}, \\
& t^{*}=\limsup _{k \longrightarrow \infty} \frac{\log n_{1} n_{2}, \ldots, n_{k+1}}{-\log c_{1} c_{2}, \ldots, c_{k}+\log n_{k+1}} .
\end{aligned}
$$

Our main results are stated as follows.

Theorem 1. Let $E \in \mathcal{J}^{*}\left(I, J,\left\{n_{k}\right\},\left\{m_{k}\right\},\left\{c_{k}\right\},\left\{\eta_{k, l}^{*}\right\}\right)$ with $\eta_{k, 0}^{*}=\eta_{k, m_{k}}^{*}=0$. For any $k \geq 1, \sigma \in D_{k-1}$, and $1 \leq j \leq m_{k}$, there exists a positive integer $\alpha$ such that $\sharp\left\{i: I_{\sigma * i} \subset J_{\sigma * j}\right\} \leq \alpha$ for the $k$-order connected component $J_{\sigma * j}$. If there exist positive constants $\tilde{c_{1}}, \tilde{c_{2}}\left(\tilde{c_{2}} \geq 1\right)$ such that at least one of the following two conditions is satisfied for any $k \geq 1$ :

(A) $\max _{1 \leq l \leq m_{k}-1} \eta_{k, l}^{*} \leq \tilde{c_{1}} \cdot c_{1} c_{2}, \ldots, c_{k}$.

(B) $\max _{1 \leq l \leq m_{k}-1} \eta_{k, l}^{*} \leq \tilde{c_{2}} \cdot \min _{1 \leq l \leq m_{k}-1} \eta_{k, l}^{*}$; then,

$$
\operatorname{dim}_{H} E=\liminf _{k \rightarrow \infty} \frac{\log n_{1} n_{2}, \ldots, n_{k-1} m_{k}}{-\log c_{1} c_{2}, \ldots, c_{k}\left(n_{k} / m_{k}\right)}=s_{*} .
$$

Example 1. Let $E$ be a $\left\{m_{k}\right\}$-quasi-homogeneous perfect set satisfying the conditions of Theorem 1, then we have $\operatorname{dim}_{H} E=s_{*}=\liminf _{k \longrightarrow \infty}\left(\log n_{1} n_{2}, \ldots, n_{k-1} n_{k} /(-\log \right.$ $\left.\left.c_{1} c_{2}, \ldots, c_{k}\right)\right)$, which is equivalent to Theorem $1.2(1)$ in [11]. Notice that if $E \in \mathscr{J}\left(I,\left\{n_{k}\right\},\left\{c_{k}\right\},\left\{\eta_{k, l}\right\}\right)\left(0 \leq l \leq n_{k}\right)$ is a homogeneous perfect set, then $E \in E \in \mathcal{J}^{*}\left(I, J,\left\{n_{k}\right\},\left\{m_{k}\right\}\right.$, $\left.\left\{c_{k}\right\},\left\{\eta_{k, l}^{*}\right\}\right)\left(1 \leq m_{k} \leq n_{k}, 0 \leq l \leq m_{k}\right)$ and satisfies $\eta_{k, 0}^{*}=\eta_{k, m_{k}}^{*}$ $=0$ (for any $k \geq 1$ ) if $\eta_{k, 0}=\eta_{k, n_{k}}=0$. Thus, Theorem 1 
generalizes Theorem $1.2(\mathrm{~A})$ and (B) in [11] under the condition $\eta_{k, 0}=\eta_{k, n_{k}}=0$ (for any $k \geq 1$ ).

Theorem 2. Suppose $E \in \mathcal{J}^{*}\left(I, J,\left\{n_{k}\right\},\left\{m_{k}\right\},\left\{c_{k}\right\},\left\{\eta_{k, l}^{*}\right\}\right)$. If $\sup _{k \geq 1} m_{k}<\infty$, then

$$
\overline{\operatorname{dim}}_{B} E=\operatorname{dim}_{p} E=s^{*} .
$$

Example 2. Let $E$ be a $\left\{m_{k}\right\}$-quasi-homogeneous Cantor set (refer to [14]); then, $E \in \mathcal{J}^{*}\left(I, J,\left\{n_{k}\right\},\left\{m_{k}\right\},\left\{c_{k}\right\},\left\{\eta_{k, l}^{*}\right\}\right)$ and satisfies $\eta_{k, 0}^{*}=\eta_{k, m_{k}}^{*}=0$ and $\eta_{k, 1}^{*}=\cdots=\eta_{k, m_{k}-1}^{*}$, for any $k \geq 1$. Since the conclusion of Theorem 2 holds under the condition $\sup _{k \geq 1} m_{k}<\infty$, Theorem 2 generalizes Theorem 2.3 in [14].

Theorem 3. Let $E \in \mathcal{J}^{*}\left(I, J,\left\{n_{k}\right\},\left\{m_{k}\right\},\left\{c_{k}\right\},\left\{\eta_{k, l}^{*}\right\}\right)$ with $\eta_{k, 0}^{*}=\eta_{k, m_{k}}^{*}=0$ and $a_{k}=\left[n_{k} / m_{k}\right]$, for any $k \geq 1$. If there exists a positive constant $\tilde{c_{3}}$ such that, for any $k \geq 1$, there exists a sequence of nonnegative integers $\left\{N_{k}\right\}_{k \geq 1}$ with $N_{k}<a_{k}$ and $\sup _{k \geq 1} N_{k}<\infty$, satisfying that, for any $k$-order connected component $J_{\sigma * j}\left(\sigma \in D_{k-1}, 1 \leq j \leq m_{k}\right), \sharp\left\{i: I_{\sigma * i} \subset J_{\sigma * j}\right\}$ is one of $a_{k}-N_{k}, \ldots, a_{k}, \ldots, a_{k}+N_{k}$, and the following condition is satisfied:

$$
\max _{1 \leq l \leq m_{k}-1} \eta_{k, l}^{*} \leq \tilde{c_{3}} \cdot c_{1} c_{2}, \ldots, c_{k}
$$

Then,

$$
\overline{\operatorname{dim}}_{B} E=\limsup _{k \longrightarrow \infty} \frac{\log n_{1} n_{2}, \ldots, n_{k-1} m_{k}}{-\log \left(c_{1} c_{2}, \ldots, c_{k-1} / m_{k}\right)} .
$$

Remark 2. The conditions of $N_{k}$ and $\sharp\left\{i: I_{\sigma * i} \subset J_{\sigma * j}\right\}$ in Theorem 3 imply that there exist $0<\varphi_{1}, \varphi_{2}<\infty$, such that, for any $k \geq 1, \sigma \in D_{k-1}, 1 \leq i, j \leq m_{k}$, we have $\varphi_{1} \leq\left(\left|J_{\sigma * i}\right| /\left|J_{\sigma * j}\right|\right) \leq \varphi_{2}$.

Theorem 4. Let $E \in \mathcal{J}^{*}\left(I, J,\left\{n_{k}\right\},\left\{m_{k}\right\},\left\{c_{k}\right\},\left\{\eta_{k, l}^{*}\right\}\right)$ with $\eta_{k, 0}^{*}=\eta_{k, m_{k}}^{*}=0$, for any $k \geq 1$. Suppose that there exist positive constants $c_{4}, \tilde{c_{5}}, \widetilde{c_{6}}, \gamma\left(\widetilde{c_{6}} \geq 1, \gamma \geq 1\right)$ such that, for any $k \geq 1$, at least one of the following three conditions is satisfied:

(C) $\max _{1 \leq l \leq m_{k}-1} \eta_{k, l}^{*} \leq \tilde{c}_{4} \cdot c_{1} c_{2}, \ldots, c_{k}$, and $\left(n_{k} / m_{k}\right) \leq \gamma$.

(D) $m_{k} \cdot \min _{1 \leq l \leq m_{k}-1} \eta_{k, l}^{*} \geq \tilde{c_{5}} \cdot c_{1} c_{2}, \ldots, c_{k-1}$.

(E) $\max _{1 \leq l \leq m_{k}-1} \eta_{k, l}^{*} \leq \tilde{c_{6}} \cdot \min _{1 \leq l \leq m_{k}-1} \eta_{k, l}^{*}$ and $\left(n_{k} / m_{k}\right) \leq$ $\gamma$; then,

$$
\overline{\operatorname{dim}}_{B} E=\limsup _{k \longrightarrow \infty} \frac{\log n_{1} n_{2}, \ldots, n_{k-1} m_{k}}{-\log \left(c_{1} c_{2}, \ldots, c_{k-1} / m_{k}\right)}
$$

Example 3. We obtain the following conclusions about the relationships between theorems in this paper and Theorem 1.4 in [12]:

(i) If Theorem 1.4 (A) in [12] holds for $E$ which satisfies $\eta_{k, 0}=\eta_{k, n_{k}}=0$ (for any $k \geq 1$ ) and there exists $\gamma \geq 1$ such that $\left(n_{k} / m_{k}\right) \leq \gamma($ for any $k \geq 1)$ or Theorem 1.4 (B) or (C) holds for $E$ (it means $\left(n_{k} / m_{k}\right)=1$ ) which satisfies $\eta_{k, 0}=\eta_{k, n_{k}}=0$, then we have $E \in \mathscr{J}^{*}\left(I, J,\left\{n_{k}\right\},\left\{m_{k}\right\},\left\{c_{k}\right\},\left\{\eta_{k, l}^{*}\right\}\right)\left(1 \leq m_{k} \leq n_{k}, 0 \leq\right.$ $\left.l \leq m_{k}\right)$ and satisfies the conditions of Theorem 4, then (7) holds for $E$. Since $\eta_{k, 0}=\eta_{k, n_{k}}=0$ and $m_{k} \leq n_{k} \leq \gamma m_{k}$, (7) is equivalent to (1.4) in [12] for the upper box dimension.

(ii) If Theorem 1.4 (D) in [12] holds for $E$ which satisfies $\eta_{k, 0}=\eta_{k, n_{k}}=0($ for any $k \geq 1)$, then $\sup _{k \geq 1} m_{k}<$ $\sup _{k \geq 1} n_{k}<\infty$. Thus, $E \in \mathcal{J}^{*}\left(I, J,\left\{n_{k}\right\},\left\{m_{k}\right\},\left\{c_{k}\right\}\right.$, $\left.\left\{\eta_{k, l}^{*}\right\}\right)\left(1 \leq m_{k} \leq n_{k}, 0 \leq l \leq m_{k}\right)$ and satisfies the conditions of Theorem 2; then, (4) holds for $E$. Since $\sup _{k \geq 1} n_{k}<\infty$, we have $s^{*}=t^{*}$ and (4) is equivalent to (1.4) in [12].

By the argument above, Theorems 2 and 4 generalize Theorem 1.4 in [12] for the upper box dimension under the conditions $\eta_{k, 0}=\eta_{k, n_{k}}=0$ and $\left(n_{k} / m_{k}\right) \leq \gamma($ for any $k \geq 1)$ (notice that if $E$ satisfies (B) or (C) or (D) of Theorem 1.4 in [12], we only need the condition $\left.\eta_{k, 0}=\eta_{k, n_{k}}=0\right)$.

Remark 3. The conditions of Theorem 3 and the conditions of Theorem 4 (C) do not contain each other.

If $E$ satisfies the conditions of Theorem 3, such that $m_{k} \equiv 2, \quad N_{k} \equiv 1, \quad$ and $\sup _{k \geq 1} n_{k}=\infty$, then $a_{k}=$ $\left[n_{k} / m_{k}\right] \longrightarrow \infty$ as $n_{k} \longrightarrow \infty$, and $E$ does not satisfy the conditions of Theorem 4 (C).

If $E$ satisfies the conditions of Theorem $4(\mathrm{C})$, such that $m_{k}=2 k$ and $n_{k}=3 k$ for any $k \geq 1$ (it means $\left.\left(n_{k} / m_{k}\right) \equiv(3 / 2)\right)$ and there exists a positive integer $k_{0}$ such that, for any $k \geq k_{0}$ and any $\sigma \in D_{k-1}$, the numbers of the $k$-order basic intervals contained in $J_{\sigma * 1}, J_{\sigma * 2}, \ldots, J_{\sigma * m_{k}}$ are $k+1,1,1, \ldots, 1$, then $E$ does not satisfy the conditions of Theorem 3 .

Remark 4. The upper box dimension in Theorems 3 or 4 is different from $s^{*}$ and $t^{*}$.

If $E$ satisfies the conditions of Theorem 3, such that $n_{1}=m_{1}=2, \quad n_{k}=2^{n_{1} n_{2}, \ldots, n_{k-1}}($ for any $k \geq 2$ ), $m_{k}=2^{n_{k-1}}$ (for any $\left.k \geq 2\right), a_{1}=\left[n_{1} / m_{1}\right]=1, a_{k}=\left[n_{k} / m_{k}\right]=$ $2^{n_{k-1}\left(n_{1} n_{2}, \ldots, n_{k-2}-1\right)}$ (for any $\left.k \geq 2\right), N_{k} \equiv 0$, and $c_{k}=2^{-n_{k}}$ (for any $k \geq 1$ ) or if $E$ satisfies the conditions of Theorem 4 (D) such that $n_{1}=m_{1}=2, \quad n_{k}=2^{n_{1} n_{2}, \ldots, n_{k-1}}$ (for any $k \geq 2$ ), $m_{k}=2^{n_{k-1}}($ for any $k \geq 2)$, and $c_{k}=2^{-n_{k}}($ for any $k \geq 1)$, then 


$$
\begin{aligned}
\overline{\operatorname{dim}}_{B} E & =\limsup _{k \longrightarrow \infty} \frac{1+n_{1}+n_{1} n_{2}+\cdots+n_{1}, \ldots, n_{k-2}+n_{k-1}}{n_{1}+n_{2}+\cdots+n_{k-1}+n_{k-1}}=\frac{1}{2}, \\
s^{*} & =\limsup _{k \longrightarrow \infty} \frac{1+n_{1}+n_{1} n_{2}+\cdots+n_{1}, \ldots, n_{k-2}+n_{1}, \ldots, n_{k-2} n_{k-1}}{n_{1}+n_{2}+\cdots+n_{k-1}+n_{k}}=0, \\
t^{*} & =\limsup _{k \longrightarrow \infty} \frac{1+n_{1}+n_{1} n_{2}+\cdots+n_{1}, \ldots, n_{k-2}+n_{1}, \ldots, n_{k-1}+n_{1}, \ldots, n_{k}}{n_{1}+n_{2}+\cdots+n_{k-1}+n_{k}+n_{1}, \ldots, n_{k}}=1 .
\end{aligned}
$$

It can be concluded that $\overline{\operatorname{dim}}_{B} E$ is different from $s^{*}$ and $t^{*}$.

\section{Proofs of the Theorems}

We shall use the following lemmas to prove the theorems.

Lemma 1 (mass distribution principle, (see [10]). Let $E$ be a Borel set and $\mu$ be a Borel measure supported on E such that $0<\mu(E)<\infty$. Suppose that, for some $s$, there are numbers $c>0$ and $\delta>0$ such that $\mu(U) \leq c|U|^{s}$, for all sets $U$ with $|U| \leq \delta$. Then, $\operatorname{dim}_{H} E \geq s$.

Lemma 2 (see [13]). Let $E \in \mathcal{J}^{*}\left(I, J,\left\{n_{k}\right\},\left\{m_{k}\right\}\right.$, $\left.\left\{c_{k}\right\},\left\{\eta_{k, l}^{*}\right\}\right)$; then,

$$
\operatorname{dim}_{H} E \leq \liminf _{k \rightarrow \infty} \frac{\log n_{1} n_{2}, \ldots, n_{k-1} m_{k}}{-\log c_{1} c_{2}, \ldots, c_{k}\left(n_{k} / m_{k}\right)}
$$

Lemma 3 (see [8]). Let $E \in \mathscr{M}\left(I,\left\{n_{k}\right\},\left\{c_{k}\right\}\right)$, then

$$
s^{*} \leq \operatorname{dim}_{p} E \leq \overline{\operatorname{dim}}_{B} E \leq t^{*} .
$$

Lemma 4 (see [15]) Let $E \in \mathcal{J}^{*}\left(I, J,\left\{n_{k}\right\},\left\{m_{k}\right\},\left\{c_{k}\right\},\left\{\eta_{k, l}^{*}\right\}\right)$; then,

$$
\overline{\operatorname{dim}}_{B} E=\inf \left\{s>0: \sum_{k=1}^{\infty} n_{1} n_{2} \cdots n_{k} \sum_{i=0}^{m_{k+1}}\left(\eta_{k+1, i}^{*}\right)^{s}<\infty\right\} .
$$

4.1. Proof of Theorem 1. Since the conditions of Theorem 1 imply that $\left(n_{k} / m_{k}\right) \leq \alpha$, for all $k \geq 1$, we have $\liminf _{k \rightarrow \infty}\left(\log n_{1} n_{2}, \ldots, n_{k-1} m_{k} /-\log c_{1} c_{2}, \ldots, \quad c_{k}\left(n_{k} /\right.\right.$ $\left.\left.m_{k}\right)\right)=\liminf _{k \longrightarrow \infty}\left(\log n_{1} n_{2}, \ldots, n_{k-1} n_{k} /-\log c_{1} c_{2}, \ldots\right.$, $\left.c_{k}\right)=s_{*}$. We only need to prove $\operatorname{dim}_{H} E=\liminf _{k \longrightarrow \infty}$ $\left(\log n_{1} n_{2}, \ldots, n_{k-1} m_{k} /-\log c_{1} c_{2}, \ldots, c_{k}\left(n_{k} / m_{k}\right)\right)$.

By Lemma 2, it is suffices to prove that if $E$ satisfies the conditions of Theorem 1, then $\operatorname{dim}_{H} E=\liminf _{k \longrightarrow \infty}$ $\left(\log n_{1} n_{2}, \ldots, n_{k-1} m_{k} /-\log c_{1} c_{2}, \ldots, c_{k}\left(n_{k} / m_{k}\right)\right)$.

For convenience, let

$$
\begin{aligned}
& \delta_{k}=c_{1} c_{2}, \ldots, c_{k}, \\
& \bar{\alpha}_{k}=\max _{1 \leq l \leq m_{k}-1} \eta_{k, l}^{*}, \\
& \underline{\alpha}_{k}=\min _{1 \leq l \leq m_{k}-1} \eta_{k, l}^{*}, \quad(\text { for any } k \geq 1) .
\end{aligned}
$$

For any $0<l<\liminf _{k \rightarrow \infty}\left(\log n_{1} n_{2}, \ldots, n_{k-1} m_{k} /\right.$ $\left.-\log c_{1} c_{2}, \ldots, c_{k}\left(n_{k} / m_{k}\right)\right)$, there exists a positive integer $K$ such that, for any $k \geq K$, we have

$$
n_{1} n_{2}, \ldots, n_{k-1} m_{k}\left(\delta_{k} \alpha\right)^{l} \geq n_{1} n_{2}, \ldots, n_{k-1} m_{k}\left(\delta_{k} \frac{n_{k}}{m_{k}}\right)^{l} \geq 1 .
$$

Let $\mu$ be a Borel probability measure supported on $E$, such that, for each $k$-order basic intervals $I_{\sigma * i}(k \geq 1$, $\left.\sigma \in D_{k-1}, 1 \leq i \leq n_{k}\right), \mu\left(I_{\sigma * i}\right)=\left(n_{1} n_{2}, \ldots, n_{k-1} n_{k}\right)^{-1}$. Then, for each $k$-order connected component $J_{\sigma * j}\left(1 \leq j \leq m_{k}\right)$, $\mu\left(J_{\sigma * j}\right) \leq\left(\alpha / n_{1} n_{2}, \ldots, n_{k-1} n_{k}\right) \leq\left(\alpha / n_{1} n_{2}, \ldots, n_{k-1} m_{k}\right)$.

Let $U$ be an interval with $0<|U|=\delta<\delta_{K}$; then, there exists a positive integer $k>K$ such that $\alpha \delta_{k} \leq \delta<\alpha \delta_{k-1}$. Thus, the number of the $(k-1)$-order basic intervals that intersect $U$ is at most $2 \alpha \leq 2 \alpha^{2}$. Denote by $g$ the number of the $k$-order connected components that intersect $U$. In order to estimate $\mu(U)$, we divide our proof into the following two cases:

Case (i): $\alpha \delta_{k} \leq \delta<\underline{\alpha}_{k}$.

It is obvious that $g \leq 2$. Then,

$$
\mu(U) \leq \frac{2 \alpha}{n_{1} n_{2}, \ldots, n_{k-1} m_{k}} \leq 2 \alpha\left(\delta_{k} \alpha\right)^{l} \leq 2 \alpha \delta^{l}=2 \alpha|U|^{l} .
$$

Case (ii): $\max \left\{\alpha \delta_{k}, \underline{\alpha}_{k}\right\} \leq \delta<\alpha \delta_{k-1}$

We discuss separately according to the two conditions (A) and (B) of Theorem 1.

Condition (A): $\bar{\alpha}_{k} \leq \widetilde{c_{1}} c_{1} c_{2}, \ldots, c_{k}$. Then, we have

$$
\begin{aligned}
\delta_{k-1} & =\sum_{i=1}^{m_{k}-1} \eta_{k, i}^{*}+n_{k} \delta_{k} \leq\left(m_{k}-1\right) \bar{\alpha}_{k}+n_{k} \delta_{k} \\
& \leq \tilde{c_{1}}\left(m_{k}-1\right) \delta_{k}+n_{k} \delta_{k} \leq \tilde{c_{1}} m_{k} \delta_{k}+n_{k} \delta_{k} \leq\left(\tilde{c_{1}}+1\right) n_{k} \delta_{k} .
\end{aligned}
$$

On the one hand, since the number of the $(k-1)$-order basic intervals that intersect $U$ is at most $2 \alpha^{2}$, we have $g \leq 2 \alpha^{2} m_{k}$. On the other hand, the number of the $k$-order connected components that intersect $U$ is at 
most $\left(\delta / \delta_{k}\right)+2 \leq 2\left(\left(\delta / \delta_{k}\right)+1\right) \leq\left(4 \delta / \delta_{k}\right)$. Then, by (13) and (15), we have

$$
\begin{aligned}
\mu(U) & \leq \frac{\alpha}{n_{1} n_{2}, \ldots, n_{k-1} m_{k}} \cdot \min \left\{2 \alpha^{2} m_{k}, \frac{4 \delta}{\delta_{k}}\right\} \\
& \leq \frac{\alpha}{n_{1} n_{2}, \ldots, n_{k-1} m_{k}}\left(2 \alpha^{2} m_{k}\right)^{1-l}\left(\frac{4 \delta}{\delta_{k}}\right)^{l} \\
& \leq 8 \alpha \delta^{l} \frac{1}{n_{1} n_{2}, \ldots, n_{k-1} m_{k}} m_{k}^{1-l}\left(\frac{1}{\delta_{k}}\right)^{l} \alpha^{2-2 l} \\
& \leq 8 \alpha \delta^{l} \frac{1}{n_{1} n_{2}, \ldots, n_{k-1} m_{k} \alpha^{l}} n_{k}\left(\frac{1}{n_{k} \delta_{k}}\right)^{l} \alpha^{2-l} \\
& \leq 8 \alpha^{3} \delta^{l} \frac{1}{n_{1} n_{2}, \ldots, n_{k-1} \alpha^{l}} \frac{n_{k}}{m_{k}}\left(\tilde{c_{1}}+1\right)^{l}\left(\frac{1}{\delta_{k-1}}\right)^{l} \\
& \leq 8 \alpha^{4} \delta^{l} \frac{1}{n_{1} n_{2}, \ldots, n_{k-2}\left(\delta_{k-1} \alpha\right)^{l}} \frac{1}{n_{k-1}}\left(\tilde{c_{1}}+1\right)^{l} \\
& \leq 8 \alpha^{4}\left(\tilde{c_{1}}+1\right)|U|^{l} . \\
& \leq 8 \alpha^{4}\left(\tilde{c_{1}}+1\right)^{l} \delta^{l} \frac{m_{k-1}}{n_{k-1}} \\
& \\
& \\
&
\end{aligned}
$$

Condition (B): $\bar{\alpha}_{k} \leq \tilde{c}_{2} \underline{\alpha}_{k}$. In this case, we have

$$
\begin{aligned}
\delta_{k-1} & \leq\left(m_{k}-1\right) \bar{\alpha}_{k}+n_{k} \delta_{k} \leq \tilde{c_{2}} m_{k} \underline{\alpha}_{k}+\alpha m_{k} \delta_{k} \\
& =m_{k}\left(\tilde{c_{2}} \underline{\alpha}_{k}+\alpha \delta_{k}\right) \leq m_{k} \tilde{c_{2}}\left(\underline{\alpha_{k}}+\alpha \delta_{k}\right) \\
& \leq 2 \widetilde{c_{2}} m_{k} \max \left\{\alpha \delta_{k}, \underline{\alpha}_{k}\right\} .
\end{aligned}
$$

On the one hand, $g \leq 2 \alpha^{2} m_{k}$. On the other hand, by the definition of $g$, we have

$$
\begin{aligned}
& (g-2) \delta_{k} \leq \delta \\
& (g-1) \underline{\alpha}_{k} \leq \delta .
\end{aligned}
$$

Then,

$$
g \leq \frac{\delta}{\max \left\{\delta_{k}, \underline{\alpha}_{k}\right\}}+2 \leq \frac{4 \delta}{\max \left\{\delta_{k}, \underline{\alpha}_{k}\right\}} \leq \frac{4 \delta \alpha}{\max \left\{\alpha \delta_{k}, \underline{\alpha}_{k}\right\}} .
$$

Combining (13), (17), and (19), we have

$$
\begin{aligned}
\mu(U) & \leq \frac{\alpha}{n_{1} n_{2}, \ldots, n_{k-1} m_{k}} \cdot \min \left\{2 \alpha^{2} m_{k}, \frac{4 \delta \alpha}{\max \left\{\alpha \delta_{k}, \underline{\alpha}_{k}\right\}}\right\} \\
& \leq \frac{\alpha}{n_{1} n_{2}, \ldots, n_{k-1} m_{k}}\left(2 \alpha^{2} m_{k}\right)^{1-l}\left(\frac{4 \delta \alpha}{\max \left\{\alpha \delta_{k}, \underline{\alpha}_{k}\right\}}\right)^{l} \\
& \leq 8 \delta \frac{\alpha}{\delta_{1} n_{2}, \ldots, n_{k-1} m_{k}} \alpha^{2-2 l} m_{k}^{1-l}\left(\frac{2 \tilde{c_{2}} m_{k}}{\delta_{k-1}}\right)^{l} \alpha^{l} \\
& \leq 16 \tilde{c_{2}} \alpha^{3} \delta^{l} \frac{1}{n_{1} n_{2}, \ldots, n_{k-2} n_{k-1}\left(\delta_{k-1} \alpha\right)^{l}} \\
& \leq 16 \tilde{c_{2}} \alpha^{3} \delta^{l} \frac{1}{n_{1} n_{2}, \ldots, n_{k-2} m_{k-1}\left(\delta_{k-1} \alpha\right)^{l}} \\
& \leq 16 \tilde{c_{2}} \alpha^{3} \delta^{l} \\
& =16 \tilde{c_{2}} \alpha^{3}|U|^{l} .
\end{aligned}
$$

By (14), (16), (20), and Lemma 1, we have $\operatorname{dim}_{H} E \geq l$. By the arbitrariness of $l$, we obtain $\operatorname{dim}_{H} E \geq \liminf _{k \longrightarrow \infty}$ $\left(\log n_{1} n_{2}, \ldots, n_{k-1} m_{k} /-\log c_{1} c_{2}, \ldots, c_{k}\left(n_{k} / m_{k}\right)\right)$ and complete the proof of Theorem 1 .

4.2. Proof of Theorem 2. By Lemma 3, it is suffices to prove $\overline{\operatorname{dim}}_{B} E \leq s^{*}$. The conclusion is obvious if $s^{*}=1$. Now, we assume $s^{*}<1$. For any $1>t>s^{*}=\limsup _{k \rightarrow \infty}$ $\left(\log n_{1} n_{2}, \ldots, n_{k} /-\log c_{1} c_{2}, \ldots, c_{k}\right)$, there exist $\varepsilon>0, k_{1}>0$ such that, for any $k \geq k_{1}$,

$$
t>\frac{\log n_{1} n_{2}, \ldots, n_{k}}{-\log c_{1} c_{2}, \ldots, c_{k}}+\varepsilon .
$$

Let $\quad b_{k}=t-\left(\log n_{1} n_{2}, \ldots, n_{k} /-\log c_{1} c_{2}, \ldots, c_{k}\right)>\varepsilon ;$ then, $n_{1} n_{2}, \ldots, n_{k}\left(\delta_{k}\right)^{t}=\left(\delta_{k}\right)^{b_{k}}$. By the definitions of $n_{k}$ and $c_{k}$, we have $\delta_{k}=c_{1} c_{2}, \ldots, c_{k} \leq 2^{-k}$. Since $m_{k} \geq 1$, for any $k \geq 1$, we have $m_{k}+1 \geq 2$ and $m_{k}+1 \leq 2 m_{k}$. By the convexity of $x^{s}(0<s<1)$, we obtain that

$$
\begin{aligned}
\sum_{i=0}^{m_{k+1}}\left(\eta_{k+1, i}^{*}\right)^{t} & \leq\left(m_{k+1}+1\right)\left(\frac{\sum_{i=0}^{m_{k+1}} \eta_{k+1, i}^{*}}{m_{k+1}+1}\right)^{t} \\
& =\left(m_{k+1}+1\right)\left(\frac{\left(1-c_{k+1} n_{k+1}\right) \delta_{k}}{m_{k+1}+1}\right)^{t} .
\end{aligned}
$$

Let $m=\sup _{k \geq 1} m_{k}<\infty$; by (21) and (22), we have 


$$
\begin{aligned}
\sum_{k=k_{1}}^{\infty} n_{1} n_{2}, \ldots, n_{k} \sum_{i=0}^{m_{k+1}}\left(\eta_{k+1, i}^{*}\right)^{t} & \leq \sum_{k=k_{1}}^{\infty} n_{1} n_{2}, \ldots, n_{k}\left(m_{k+1}+1\right)\left(\frac{\left(1-c_{k+1} n_{k+1}\right) \delta_{k}}{m_{k+1}+1}\right)^{t} \\
& \leq 2 m \sum_{k=k_{1}}^{\infty} n_{1} n_{2}, \ldots, n_{k}\left(\frac{\delta_{k}}{m_{k+1}+1}\right)^{t} \\
& =2 m \sum_{k=k_{1}}^{\infty} n_{1} n_{2}, \ldots, n_{k}\left(\delta_{k}^{t}\right)\left(m_{k+1}+1\right)^{-t} \\
& =2 m \sum_{k=k_{1}}^{\infty}\left(\delta_{k}^{b_{k}}\right)\left(m_{k+1}+1\right)^{-t} \\
& \leq 2 m \sum_{k=k_{1}}^{\infty}\left(\delta_{k}^{b_{k}}\right) 2^{-t} \\
& \leq 2 m \sum_{k=k_{1}}^{\infty} 2^{-k \varepsilon} \cdot 1<\infty .
\end{aligned}
$$

By Lemma 4, we have $\overline{\operatorname{dim}}_{B} E \leq t$. By the arbitrariness of $t$, we have $\overline{\operatorname{dim}}_{B} E \leq s^{*}$ and complete the proof of Theorem 1 .

4.3. Proof of Theorem 3. First, we use Lemma 4 to estimate the upper bound for $\overline{\operatorname{dim}}_{B} E$.

Proposition 1. Suppose $E \in \mathcal{J}^{*}\left(I, J,\left\{n_{k}\right\},\left\{m_{k}\right\},\left\{c_{k}\right\},\left\{\eta_{k, l}^{*}\right\}\right)$; then,

$$
\overline{\operatorname{dim}}_{B} E \leq \limsup _{k \longrightarrow \infty} \frac{\log n_{1} n_{2}, \ldots, n_{k-1} m_{k}}{-\log \left(c_{1} c_{2}, \ldots, c_{k-1} / m_{k}\right)} .
$$

Proof. For any $1>p>\limsup _{k \rightarrow \infty}\left(\log n_{1} n_{2}, \ldots, n_{k} m_{k+1} /\right.$ $\left.-\log \left(c_{1} c_{2}, \ldots, c_{k} / m_{k+1}\right)\right)$, there exist $\xi>0$ and $k_{2}>0$ such that, for any $k \geq k_{2}$,

$$
p>\frac{\log n_{1} n_{2}, \ldots, n_{k} m_{k+1}}{-\log \left(c_{1} c_{2}, \ldots, c_{k} / m_{k+1}\right)}+\xi,
$$

$$
(\xi-p) \log \frac{c_{1} c_{2}, \ldots, c_{k}}{m_{k+1}}>\log n_{1} n_{2}, \ldots, n_{k} m_{k+1},
$$

which implies

$$
n_{1} n_{2}, \ldots, n_{k} m_{k+1}<\left(\frac{c_{1} c_{2}, \ldots, c_{k}}{m_{k+1}}\right)^{\xi-p}
$$

By $\delta_{k}=c_{1} c_{2}, \ldots, c_{k} \leq 2^{-k}$, for any $k \geq 0$, and the convexity of $x^{s}(0<s<1)$, we have

$$
\begin{aligned}
\sum_{i=0}^{m_{k+1}}\left(\eta_{k+1, i}^{*}\right)^{p} & \leq\left(m_{k+1}+1\right)\left(\frac{\sum_{i=0}^{m_{k+1}} \eta_{k+1, i}^{*}}{m_{k+1}+1}\right)^{p} \\
& =\left(m_{k+1}+1\right)\left(\frac{\left(1-c_{k+1} n_{k+1}\right) \delta_{k}}{m_{k+1}+1}\right)^{p} .
\end{aligned}
$$

Since $m_{k} \geq 1$ (for any $k \geq 1$ ), we have $m_{k}+1 \leq 2 m_{k}$ for any $k \geq 1$. Then, by (27) and (28), we have

and it follows that

$$
\begin{aligned}
\sum_{k=k_{2}}^{\infty} n_{1} n_{2}, \ldots, n_{k} \sum_{i=0}^{m_{k+1}}\left(\eta_{k+1, i}^{*}\right)^{p} & \leq \sum_{k=k_{2}}^{\infty} n_{1} n_{2}, \ldots, n_{k}\left(m_{k+1}+1\right)\left(\frac{\left(1-c_{k+1} m_{k+1}\right) \delta_{k}}{m_{k+1}+1}\right)^{p} \\
& \leq 2 \sum_{k=k_{2}}^{\infty} n_{1} n_{2}, \ldots, n_{k} m_{k+1}\left(\frac{\delta_{k}}{m_{k+1}+1}\right)^{p} \\
& \leq 2 \sum_{k=k_{2}}^{\infty}\left(\frac{\delta_{k}}{m_{k+1}}\right)^{\xi-p}\left(\frac{\delta_{k}}{m_{k+1}}\right)^{p} \\
& =2 \sum_{k=k_{2}}^{\infty}\left(\frac{\delta_{k}}{m_{k+1}}\right)^{\xi} \\
& \leq 2 \sum_{k=k_{2}}^{\infty} 2^{-k \xi}<\infty
\end{aligned}
$$


By Lemma 4 , we have $\overline{\operatorname{dim}}_{B} E \leq p$. By the arbitrariness of $p$, we have $\overline{\operatorname{dim}}_{B} E \leq \limsup \operatorname{lom}_{k \rightarrow \infty}\left(\log n_{1} n_{2}, \ldots, n_{k-1} m_{k} /\right.$ $\left.-\log \left(c_{1} c_{2}, \ldots, c_{k-1} / m_{k}\right)\right)$.

Now, we estimate the lower bound for $\overline{\operatorname{dim}}_{B} E$.

Proposition 2. Suppose $E \in \mathcal{J}^{*}\left(I, J,\left\{n_{k}\right\},\left\{m_{k}\right\},\left\{c_{k}\right\},\left\{\eta_{k, l}^{*}\right\}\right)$ satisfies the conditions of Theorem 3; then,

$$
\overline{\operatorname{dim}}_{B} E \geq \limsup _{k \longrightarrow \infty} \frac{\log n_{1} n_{2}, \ldots, n_{k-1} m_{k}}{-\log \left(c_{1} c_{2}, \ldots, c_{k-1} / m_{k}\right)} .
$$

Proof. Let $r_{1}=\left(1 / m_{1}\right)$ and $r_{k}=\left(c_{1} c_{2}, \ldots, c_{k-1} / m_{k}\right)$ (for any $k \geq 2)$. We estimate $\left(\log N_{r_{k}}(E) /-\log r_{k}\right)$ for a fixed $k$ large enough.

By the condition of Theorem 3, we have $\bar{\alpha}_{k} \leq \tilde{c}_{3} \cdot c_{1} c_{2}, \ldots, c_{k}$. Let $M_{k}$ be the maximum number of the $k$-order connected components intersecting a ball of diameter $r_{k}$; then, we have

$$
\begin{aligned}
\left(M_{k}-2\right)\left(a_{k}-N_{k}\right) c_{1} c_{2}, \ldots, c_{k} & \leq r_{k}=\frac{c_{1} c_{2}, \ldots, c_{k-1}}{m_{k}} \\
& \leq \frac{n_{k} c_{1} c_{2}, \ldots, c_{k}+\left(m_{k}-1\right) \bar{\alpha}_{k}}{m_{k}} \leq \frac{n_{k} c_{1} c_{2}, \ldots, c_{k}+\left(m_{k}-1\right) \tilde{c_{3}} \cdot c_{1} c_{2}, \ldots, c_{k}}{m_{k}} \\
& \leq \frac{n_{k} c_{1} c_{2}, \ldots, c_{k}+\left(n_{k}-1\right) \tilde{c_{3}} \cdot c_{1} c_{2}, \ldots, c_{k}}{m_{k}} \leq \frac{c_{1} c_{2}, \ldots, c_{k}\left(n_{k}+n_{k} \tilde{c_{3}}\right)}{m_{k}},
\end{aligned}
$$

which implies

$$
M_{k}-2 \leq \frac{\left(n_{k} / m_{k}\right)\left(1+\tilde{c_{3}}\right)}{a_{k}-N_{k}} .
$$

Let $N=\sup _{k \geq 1} N_{k}$, then we have $\left(\left(n_{k} / m_{k}\right) / a_{k}-\right.$ $\left.N_{k}\right) \leq\left(\left(a_{k}+N_{k}\right) / a_{k}-N_{k}\right)=1+\left(2 N_{k} /\left(a_{k}-N_{k}\right)\right) \leq 1+2$ $N_{k} \leq 2 N+1$. Then, $M_{k} \leq 2+\left(1+\tilde{c_{3}}\right)(2 N+1)$. Thus,

$$
\begin{aligned}
\frac{\log N_{r_{k}}(E)}{-\log r_{k}} & \geq \frac{\log \left(1 / M_{k}\right) n_{1} n_{2}, \ldots, n_{k-1} m_{k}}{-\log r_{k}} \\
& \geq \frac{\log n_{1} n_{2}, \ldots, n_{k-1} m_{k}-\log \left(2+\left(1+\widetilde{c_{3}}\right)(2 N+1)\right)}{-\log \left(c_{1} c_{2}, \ldots, c_{k-1} / m_{k}\right)} .
\end{aligned}
$$

Since $r_{k} \longrightarrow 0$ as $k \longrightarrow \infty$, then, by (33), we have

$$
\begin{aligned}
\overline{\operatorname{dim}}_{B} E & =\limsup _{k \longrightarrow \infty} \frac{\log N_{\varepsilon}(E)}{-\log \varepsilon} \geq \limsup _{k \longrightarrow \infty} \frac{\log N_{r_{k}}(E)}{-\log r_{k}} \\
& \geq \limsup _{k \longrightarrow \infty} \frac{\log n_{1} n_{2}, \ldots, n_{k-1} m_{k}}{-\log \left(c_{1} c_{2}, \ldots, c_{k-1} / m_{k}\right)} .
\end{aligned}
$$

From Propositions 1 and 2, we have Theorem 3.

4.4. Proof of Theorem 4. It is obvious that the conclusion of Proposition 1 also holds if $E$ satisfies the conditions of Theorem 4, then it is suffices to estimate the lower bound of $\overline{\operatorname{dim}}_{B} E$ under the conditions of Theorem 4 .

Proposition 3. Suppose $E \in \mathcal{J}^{*}\left(I, J,\left\{n_{k}\right\},\left\{m_{k}\right\},\left\{c_{k}\right\},\left\{\eta_{k, l}^{*}\right\}\right)$ satisfies the conditions of Theorem 4; then,

$$
\overline{\operatorname{dim}}_{B} E \geq \limsup _{k \longrightarrow \infty} \frac{\log n_{1} n_{2}, \ldots, n_{k-1} m_{k}}{-\log \left(c_{1} c_{2}, \ldots, c_{k-1} / m_{k}\right)} .
$$

Proof. We discuss separately according to the three conditions (C), (D), and (E) of Theorem 4 to estimate $\left(\log N_{r_{k}}(E) /-\log r_{k}\right)$ for a fixed $k$ large enough, and we use the definitions of $r_{k}$ and $M_{k}$ in the proof of Theorem 3 .

Condition (C): in this case, $\bar{\alpha}_{k} \leq \tilde{c_{4}} \cdot c_{1} c_{2}, \ldots, c_{k}$ and $\left(n_{k} / m_{k}\right) \leq \gamma$. Then, we have

$$
\begin{aligned}
\left(M_{k}-2\right) c_{1} c_{2}, \ldots, c_{k} & \leq r_{k}=\frac{c_{1} c_{2}, \ldots, c_{k-1}}{m_{k}} \\
& \leq \frac{n_{k} c_{1} c_{2}, \ldots, c_{k}+\left(m_{k}-1\right) \bar{\alpha}_{k}}{m_{k}} \leq \frac{n_{k} c_{1} c_{2}, \ldots, c_{k}+\left(m_{k}-1\right) \tilde{c_{4}} \cdot c_{1} c_{2}, \ldots, c_{k}}{m_{k}} \\
& \leq \frac{n_{k} c_{1} c_{2}, \ldots, c_{k}+\left(n_{k}-1\right) \tilde{c_{4}} \cdot c_{1} c_{2}, \ldots, c_{k}}{m_{k}} \leq \frac{c_{1} c_{2}, \ldots, c_{k}\left(n_{k}+n_{k} \tilde{c_{4}}\right)}{m_{k}},
\end{aligned}
$$


it follows

$$
M_{k}-2 \leq \frac{n_{k}+n_{k} \tilde{c_{4}}}{m_{k}} \leq \gamma\left(1+\tilde{c_{4}}\right)
$$

which implies $M_{k} \leq 2+\gamma\left(1+\tilde{c_{4}}\right)$. Thus,

$$
\begin{aligned}
\frac{\log N_{r_{k}}(E)}{-\log r_{k}} & \geq \frac{\log \left(1 / M_{k}\right) n_{1} n_{2}, \ldots, n_{k-1} m_{k}}{-\log r_{k}} \\
& \geq \frac{\log n_{1} n_{2}, \ldots, n_{k-1} m_{k}-\log \left(2+\gamma\left(1+\widetilde{c_{4}}\right)\right)}{-\log \left(c_{1} c_{2}, \ldots, c_{k-1} / m_{k}\right)} .
\end{aligned}
$$

Condition (D): in this case, $m_{k} \cdot \underline{\alpha} k \geq \widetilde{c_{5}} \cdot c_{1} c_{2}, \ldots, c_{k-1}$. Then,

$$
\underline{\alpha}_{k} \geq \frac{\tilde{c_{5}} c_{1} c_{2}, \ldots, c_{k-1}}{m_{k}}=\tilde{c_{5}} r_{k}
$$

which implies that the number of the $k$-order connected components intersecting a ball of diameter $\tilde{c_{5}} r_{k}$ is at most 2; then, the number of the $k$-order connected components intersecting a ball of diameter $r_{k}$ is at most $2\left(\left(1 / \widetilde{c_{5}}\right)+1\right):=T_{k}$. Thus,

$$
\begin{aligned}
\frac{\log N_{r_{k}}(E)}{-\log r_{k}} & \geq \frac{\log \left(1 / T_{k}\right) n_{1} n_{2}, \ldots, n_{k-1} m_{k}}{-\log r_{k}} \\
& \geq \frac{\log n_{1} n_{2}, \ldots, n_{k-1} m_{k}-\log \left(2\left(\left(1 / \widetilde{c_{5}}\right)+1\right)\right)}{\log \left(c_{1} c_{2}, \ldots, c_{k-1} / m_{k}\right)} .
\end{aligned}
$$

Condition (E): in this case, $\bar{\alpha}_{k} \leq \tilde{c_{6}} \cdot \underline{\alpha}_{k}$. We distinguish two cases.

Case 1: $\underline{\alpha}_{k}<c_{1} c_{2}, \ldots, c_{k}$; then, $\bar{\alpha}_{k} \leq \tilde{c_{6}} \cdot c_{1} c_{2}, \ldots, c_{k}$. Similar to the discussions in condition (C), we have

$$
\begin{aligned}
\frac{\log N_{r_{k}}(E)}{-\log r_{k}} & \geq \frac{\log \left(1 / M_{k}\right) n_{1} n_{2}, \ldots, n_{k-1} m_{k}}{-\log r_{k}} \\
& \geq \frac{\log n_{1} n_{2}, \ldots, n_{k-1} m_{k}-\log \left(2+\gamma\left(1+\tilde{c_{6}}\right)\right)}{-\log \left(c_{1} c_{2}, \ldots, c_{k-1} / m_{k}\right)} .
\end{aligned}
$$

Case 2: $\underline{\alpha}_{k} \geq c_{1} c_{2}, \ldots, c_{k}$. In this case, we have

$$
\begin{aligned}
c_{1} c_{2}, \ldots, c_{k-1} & \leq n_{k} c_{1} c_{2}, \ldots, c_{k}+\left(m_{k}-1\right) \bar{\alpha}_{k} \\
& \leq n_{k} c_{1} c_{2}, \ldots, c_{k}+\left(m_{k}-1\right) \tilde{c_{6}} \underline{\alpha}_{k} \\
& \leq n_{k} c_{1} c_{2}, \ldots, c_{k}+n_{k} \tilde{c_{6}} \underline{\alpha}_{k} \\
& \leq n_{k} \underline{\alpha}_{k}+n_{k} \tilde{c_{6}} \underline{\alpha}_{k},
\end{aligned}
$$

and it follows that

$$
\underline{\alpha}_{k} \geq \frac{c_{1} c_{2}, \ldots, c_{k-1}}{n_{k}\left(1+\tilde{c_{6}}\right)} \geq \frac{c_{1} c_{2}, \ldots, c_{k-1}}{\gamma\left(m_{k}\left(1+\widetilde{c_{6}}\right)\right)}=\frac{1}{\gamma\left(1+\widetilde{c_{6}}\right)} \cdot r_{k} .
$$

Similarly to the discussions in Condition (D), we have

$$
\frac{\log N_{r_{k}}(E)}{-\log r_{k}} \geq \frac{\log n_{1} n_{2}, \ldots, n_{k-1} m_{k}-\log 2\left(\gamma\left(\tilde{c_{6}}+1\right)+1\right)}{\log \left(c_{1} c_{2}, \ldots, c_{k-1} / m_{k}\right)} .
$$

Notice that $r_{k} \longrightarrow 0$ as $k \longrightarrow \infty$. Then, by (38), (40), (41), and (44), we have

$$
\begin{aligned}
\overline{\operatorname{dim}}_{B} E & =\limsup _{\varepsilon \longrightarrow 0} \frac{\log N_{\varepsilon}(E)}{-\log \varepsilon} \geq \limsup _{k \longrightarrow \infty} \frac{\log N_{r_{k}}(E)}{-\log r_{k}} \\
& \geq \limsup _{k \longrightarrow \infty} \frac{\log n_{1} n_{2}, \ldots, n_{k-1} m_{k}}{-\log \left(c_{1} c_{2}, \ldots, c_{k-1} / m_{k}\right)} .
\end{aligned}
$$

From Propositions 1 and 3, we have Theorem 4.

\section{Data Availability}

The data used to support the study are included within the article.

\section{Conflicts of Interest}

The authors declare that they have no conflicts of interest.

\section{Acknowledgments}

The authors thank Professor Wu Min of South China University of Technology for her helpful discussions and suggestions. This work was supported by the National Natural Science Foundation of China (no. 11901121), Guangxi Natural Science Foundation (2020GXNSFAA297040), special foundation for Guangxi Ba Gui Scholars, Guangdong Natural Science Foundation (2018A030313971), and The Education Department Foundation of Guangdong Province (2017KTSCX112).

\section{References}

[1] M. Lou and M. Wu, "The pointwise dimensions of Moran measures," Science China Mathematics, vol. 53, no. 5, pp. 1283-1292, 2010.

[2] A. Käenmäki, B. Li, and V. Suomala, "Local dimensions in Moran constructions," Nonlinearity, vol. 29, no. 3, pp. 807-822, 2016.

[3] J. Yang, A. Käenmäki, and M. Wu, "A note on correlation and local dimensions," Chaos, Solitons \& Fractals, vol. 97, no. 1, pp. 39-43, 2017.

[4] Y. Dai, Z. Wen, L. Xi, and Y. Xiong, "Quasisymmetrically minimal Moran sets and Hausdorff dimension," Annales Academiae Scientiarum Fennicae Mathematica, vol. 36, no. 1, pp. 139-151, 2011.

[5] Y. Li, M. Wu, and L. Xi, "Quasisymmetric minimality on packing dimension for Moran sets," Journal of Mathematical Analysis and Applications, vol. 408, no. 1, pp. 324-334, 2013.

[6] K. J. Falconer, Techniques in Fractal Geometry, John Wiley \& Sons Ltd., Chichester, UK, 1997.

[7] B.-W. Wang and J. Wu, "Hausdorff dimension of certain sets arising in continued fraction expansions," Advances in Mathematics, vol. 218, no. 5, pp. 1319-1339, 2008.

[8] D. Feng, Z. Wen, and J. Wu, "Some dimensional results for homogeneous Moran sets," Science in China Series A: Mathematics, vol. 40, no. 5, pp. 475-482, 1997. 
[9] Z. Wen, "Moran sets and Moran classes," Chinese Science Bulletin, vol. 46, no. 22, pp. 1849-1856, 2001.

[10] K. J. Falconer, Fractal Geometry: Mathematical Foundations and Applications, John Wiley \& Sons, New York, NY, USA, 1990.

[11] Z. Y. Wen and J. Wu, "Hausdorff dimension of homogeneous perfect sets," Acta Mathematica Hungarica, vol. 107, no. 12, pp. 35-44, 2005.

[12] X. Y. Wang and J. Wu, "Packing dimensions of homogeneous perfect sets," Acta Mathematica Hungarica, vol. 118, no. 12, pp. 29-39, 2007.

[13] X. Hu, "Some dimensional results for a class of special homogeneous Moran sets," Czechoslovak Mathematical Journal, vol. 66, no. 1, pp. 127-135, 2016.

[14] X. M. Hu, "Packing dimensional results for homogeneous Moran sets (in Chinese)," Acta Mathematica Scientia, vol. 36A, no. 5, pp. 873-878, 2016.

[15] C. Tricot, "Douze définitions de la densité logarithmique," $C R$ Acad Sci Paris Série I Math, vol. 293, pp. 549-552, 1981. 